\title{
Prognostic Significance of Tumor Regression Grade after Preoperative Chemoradiotherapy for Rectal Cancer
}

\author{
Byung Chun Kim \\ Department of Surgery, Kangnam Sacred Heart Hospital, Hallym University College of Medicine, Seoul, Korea
}

\section{See Article on Page 31-40}

In recent years, a number of studies on the use of preoperative chemoradiotherapy (CRT) in patients with cT3-4 rectal cancer have reported a pathologically complete response rate of 9-29\%, as well as an increased ability to perform sphincter-sparing surgery $[1,2]$. Preoperative CRT is superior to postoperative treatment in terms of local control and toxicity [3]. In the Swedish Rectal Cancer Trial, the rate of local recurrence was $27 \%$ with surgery alone, $20 \%$ with postoperative radiation (60 Gy in 8 weeks) and $11 \%$ with preoperative radiation (25 Gy in 5 fractions) [4]. There was also a significant improvement in the 5-year diseasefree survival rate, which was attributed to a lower rate of distant metastases, with preoperative radiation when compared to either surgery alone or surgery with postoperative radiation [4]. However, tumor response after preoperative CRT for rectal cancer varies considerably. Recent studies have demonstrated that good response to preoperative CRT is a favorable prognostic factor and that a high grade of tumor regression is indicative of better survival [5]. Therefore, the accurate evaluation of tumor response to preoperative CRT is suggested to be essential for predicting oncologic outcomes and for planning further treatment.

Regressive changes of the primary tumors in response to preoperative CRT were documented as described by Mandard et al. [6]. They first developed a five-point grading scale to assess the response to preoperative CRT in esophageal cancer. However, only two groups of tumor regression grades (TRGs) were prognostically relevant (grades 1, 2, 3 vs. 4, 5). Rodel et al. [5] suggested that primary tumor regression was grouped into three categories:

Correspondence to: Byung Chun Kim, M.D.

Department of Surgery, Kangnam Sacred Heart Hospital, Hallym University College of Medicine, 98-1 Daerim 1-dong, Yeongdeungpo-gu, Seoul

150-950, Korea

Tel: +82-2-829-5130, Fax: +82-2-834-6526

E-mail: bckimgs@hallym.or.kr

(C) 2011 The Korean Society of Coloproctology

This is an open-access article distributed under the terms of the Creative Commons Attribution NonCommercial License (http://creativecommons.org/licenses/by-nc/3.0) which permits unrestricted noncommercial use, distribution, and reproduction in any medium, provided the original work is properly cited.
Grade 1 (complete regression) showed an absence of histologically identifiable residual cancer and predominant fibrosis extending through the different layers of the rectal wall. Grade 2 (intermediate regression) was characterized by an increase in the number of residual cancer cells, but still fibrosis was dominant. Grade 3 (poor regression) showed residual cancer outgrowing fibrosis, a scant presence or a complete absence of regressive changes, and residual cancer cells.

In this study, the authors investigated the prognostic significance of a semi-quantitative grading system for tumor regression after preoperative CRT. First, the authors performed analyses for all patients and then for the subgroup of patients who had no lymph node metastasis (ypN0). The authors also investigated whether TRG had any correlation with the presence of metastatic lymph nodes or with histopathologic T- and N-downstagings. Finally, the authors investigated whether different prognoses were observed among ypN0 patients with different TRGs given a specific ypTN stage or vice versa. The authors suggested in this study that TRG was found to have a limited prognostic significance following preoperative chemoradiation therapy for the treatment of locally advanced rectal cancer. Although, as a whole, TRG had a weaker prognostic power than ypN stage, it was found to have the strongest prognostic power in the patients without lymph node metastasis. For better prediction of oncologic outcomes after preoperative chemoradiation therapy in locally advanced rectal cancer, tumor regression grade, in addition to $\mathrm{ypT}$ and $\mathrm{ypN}$ staging system, should be addressed.

If evaluation of TRG is to be implemented in pathologic reports, a standard method of assessing tumor response is clearly required. Although tumor regression grade basically scores the ratio of residual cancer cell to radiation-induced fibrosis, there is still no a standard method for scoring tumor regression grade. This is important because documentation of TRG may be different depending on the method of preparing slides, the number of slides reviewed per tumor, the experience of the reviewers, and so on. The three-point grade has the advantage of better reproducibility, with similar prognostic significance [5].

It is clear that there are multiple clinical and histopathological factors that are relevant in determining the prognosis after preoperative CRT. Long-term oncologic outcomes in patients with rec- 
tal cancer after preoperative CRT were found to depend on histopathologic $\mathrm{T}$ and $\mathrm{N}$ downstaging and on tumor regression grade. However, TRG alone is not definitive for giving a prognosis. Wellestablished histopathologic factors, in particular the ypT and ypN category, remain the most important prognostic factors [7]. Positive lymph nodes after preoperative CRT indicate both an aggressive potential of the malignant cells in regional lymph nodes and a resistance of those cells to CRT. Thus, traditional histopathologic staging, especially $\mathrm{N}$ staging, remains the most important prognostic factor for cancer-specific survival, and TRG may aid in determining a prognosis for patients, particularly those without lymph node metastasis. In patients who achieved $\mathrm{N}$-downstaging after preoperative chemoradiation, an accurate prediction of cancerspecific survival requires information on both the number of viable cancer cells remaining (TRG) and whether or not the foci of viable cancer cells are located within or outside the rectal wall (ypT stage).

Tumor regression grade seems to be a prognostic factor for disease-free survival in patients receiving preoperative CRT for rectal cancer. In addition, it is a prognostic factor for local failure, metastasis-free survival, and overall survival [8].

\section{REFERENCES}

1. Valentini V, Coco C, Cellini N, Picciocchi A, Genovesi D, Mantini G, et al. Preoperative chemoradiation for extraperitoneal T3 rectal cancer: acute toxicity, tumor response, and sphincter preserva- tion. Int J Radiat Oncol Biol Phys 1998;40:1067-75.

2. Janjan NA, Crane C, Feig BW, Cleary K, Dubrow R, Curley S, et al. Improved overall survival among responders to preoperative chemoradiation for locally advanced rectal cancer. Am J Clin Oncol 2001;24:107-12.

3. Sauer R, Becker H, Hohenberger W, Rodel C, Wittekind C, Fietkau R, et al. Preoperative versus postoperative chemoradiotherapy for rectal cancer. N Engl J Med 2004;351:1731-40.

4. Improved survival with preoperative radiotherapy in resectable rectal cancer. Swedish Rectal Cancer Trial. N Engl J Med 1997; 336:980-7.

5. Rodel C, Martus P, Papadoupolos T, Fuzesi L, Klimpfinger M, Fietkau R, et al. Prognostic significance of tumor regression after preoperative chemoradiotherapy for rectal cancer. J Clin Oncol 2005; 23:8688-96.

6. Mandard AM, Dalibard F, Mandard JC, Marnay J, Henry-Amar $\mathrm{M}$, Petiot JF, et al. Pathologic assessment of tumor regression after preoperative chemoradiotherapy of esophageal carcinoma. Clinicopathologic correlations. Cancer 1994;73:2680-6.

7. Chapet O, Romestaing P, Mornex F, Souquet JC, Favrel V, Ardiet JM, et al. Preoperative radiotherapy for rectal adenocarcinoma: Which are strong prognostic factors? Int J Radiat Oncol Biol Phys 2005;61:1371-7.

8. Vecchio FM, Valentini V, Minsky BD, Padula GD, Venkatraman ES, Balducci M, et al. The relationship of pathologic tumor regression grade (TRG) and outcomes after preoperative therapy in rectal cancer. Int J Radiat Oncol Biol Phys 2005;62:752-60. 